\title{
Experimental Investigation on the Ice Formation and Growth in Ex Vivo Bovine Liver
}

\author{
Valter Giaretto ${ }^{1}$, Claudio Passerone ${ }^{2, *}$ \\ ${ }^{1}$ Department of Energy, Politecnico di Torino, Torino, Italy \\ ${ }^{2}$ Department of Electronics and Telecommunications, Politecnico di Torino, Torino, Italy \\ Email address: \\ valter.giaretto a polito.it (V. Giaretto), claudio.passerone a polito.it (C. Passerone) \\ ${ }^{*}$ Corresponding author
}

To cite this article:

Valter Giaretto, Claudio Passerone. Experimental Investigation on the Ice Formation and Growth in Ex Vivo Bovine Liver. American Journal of BioScience. Vol. 6, No. 3, 2018, pp. 35-44. doi: 10.11648/j.ajbio.20180603.11

Received: September 20, 2018; Accepted: October 11, 2018; Published: November 15, 2018

\begin{abstract}
The paper presents a set of experiments to characterize the ice front propagation in ex vivo bovine liver samples. Based on previous successful experiments using water and agar-gel as tested materials, the methodology infers the ice evolution in the sample from temperature measures obtained at the cooling device only, with a technique known as mirror image. No analytical or numerical solutions are proposed for the phase change propagation inside the tested material, such as for instance the Stefan approach as a classical free boundary problem for a parabolic partial differential equation. Thermal events inside the tested material, such as the heat flux released during the phase transition, have been deduced by the measured temperatures and the numerical solution of the thermal field inside the cooling device, conceived to mimic some thermal features of an actual cryogenic probe. The application domain for these experiments is in the cryoablative therapy, and the aim is to help providing an increased knowledge on the thermal effects to better deduce the effectiveness of the treatment and reduce recurrences, while at the same time avoid damages in the proximal anatomical structures. Results show that it is possible to detect the triggering of ice formation at the interface between the liver samples and the cooling device and predict the ice front propagation according to a law linear on the heat subtracted per unit of area. In a few tens of seconds, the maximum ice penetration distance is about $2 \mathrm{~mm}$ inside the liver tissue, with a penetration rate that goes from $0.2 \mathrm{~mm} / \mathrm{s}$ to $0.02 \mathrm{~mm} / \mathrm{s}$. Moreover, adopting a special sample configured as an agar-gel coating superimposed to an ex vivo liver layer, the arrival time of the ice penetrating the liver and the temperature at the interface between these materials were detected, in order to estimate the part of the heat flux useful to the ice formation with respect to that spent for cooling the surrounding medium. Based on this preliminary result, to improve the cryoablation effectiveness it could be useful to increase the heat flux per unit of surface at the beginning, instead of the ablation duration.
\end{abstract}

Keywords: Cryoablation, Ex Vivo Liver, Mirror Image Technique, Ice formation in Tissue, Cryosurgery

\section{Introduction}

The cryoablation theme is one, among various, on which a profitable collaboration between medical doctors and engineers can be developed. Everything related to the heat exchange mechanisms and interface phenomena, the thermodynamic processes of multi-phase and multi-component systems, constitute a common ground on which to share skills and knowledge.

As known in cryoablation, whatever is the actual biological mechanisms responsible for cell death, it is the ice formation within tissues that leads to cell damage [1-3], and a wide literature is devoted to analyzing the damage on living tissues caused by the temperature level and cooling rates [4-10].

During a clinical treatment the knowledge of thermophysical quantities relevant to the cryogenic probe, such as its temperature level, heat flux and cooling rate, is not the guarantee of an effective application, but it may be useful to enhance its success. Some of these quantities are directly accessible from the equipment available to the clinician, others can be attained with well oriented technical improvements, but for a successful application the major trouble is probably the lack of knowledge of what happens just beyond the 
probe-tissue interface.

The collaboration with cardiologists of the Cardiology Division of the Department of Medical Sciences, Città della Salute e della Scienza, Hospital University of Turin, aims to understand the influence of some thermophysical quantities involving the treatment of cardiac arrhythmia [11-12, 18-19]. The assessments on physical behavior and technical aspects of cryogenic probes have been addressed to preserve the clinical requirements in reducing recurrences [20-21], increasing the thermal effectiveness on treated biological tissues, avoiding damages in the proximal anatomical structures [22-23].

According to these needs, an investigation path has been planned to deepen the knowledge on the thermophysical behavior of a biological tissue subject to freezing at the probe interface, with the aim of looking for the useful criteria for optimizing the cryogenic probe. Since the analysis of heat exchange at the inner and outer interfaces of the cryogenic probe is seen as a key step to drive efforts toward the expected clinical improvements, different experimental approaches obtained with various apparatuses have been considered.

The inspection of the inner probe interface requires to reproduce an experimental context very close to that of a real cryoprobe, in which the freezing criterion and some probe features (materials, shape, etc.) must be preserved. On the contrary, in the analysis of the outer interface, tested material and outside boundary conditions assume a main role, while the shape of the probe and the way in which the freezing effect is produced appear at first less important.

The outer interface has been first investigated employing

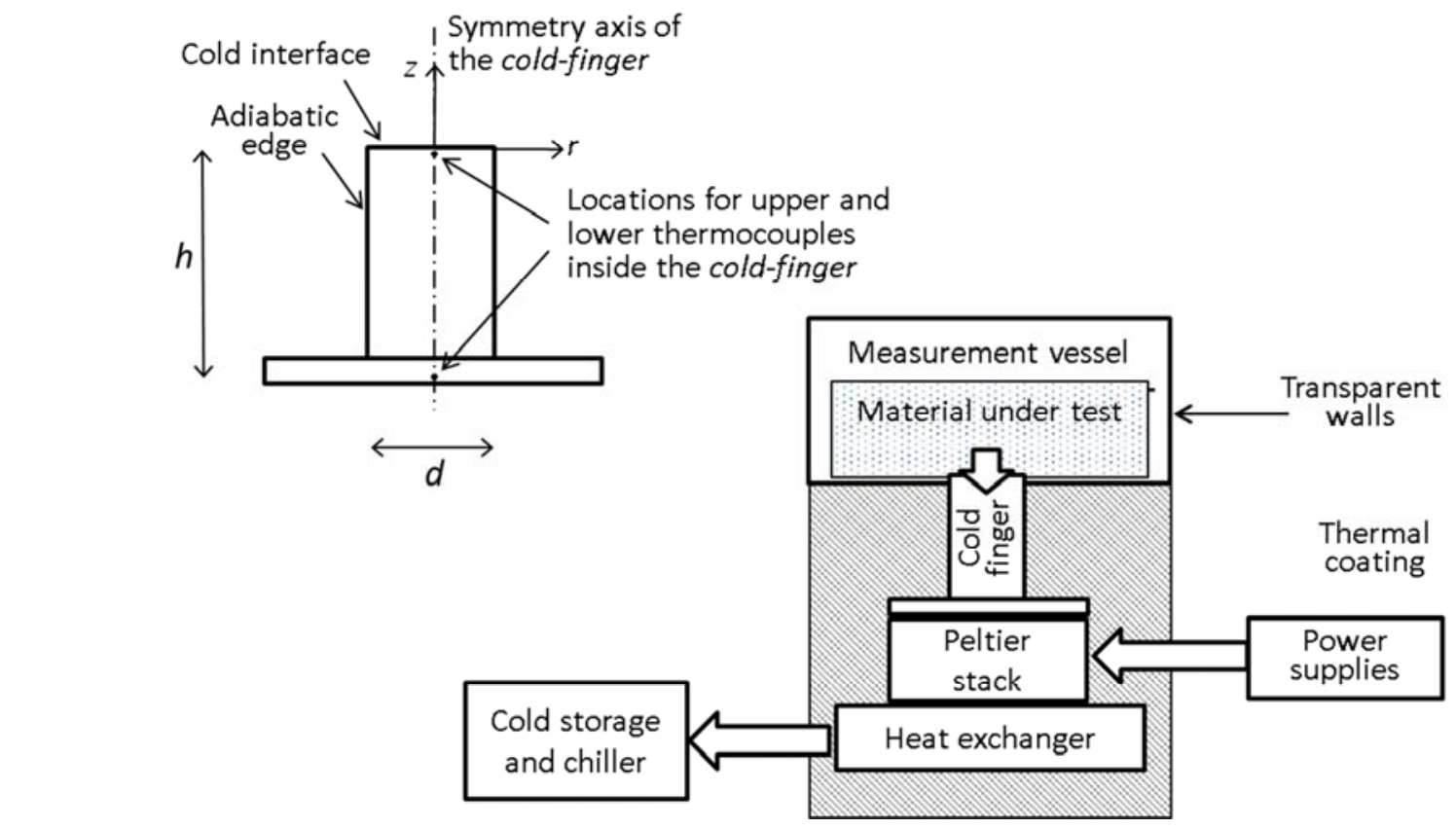

the experimental setup already described and validated by Giaretto and Passerone, adopting the experimental methodology summarized in the next section [13]. The preliminary tests performed using water and agar-gel showed, among others, some relevant results, for which the beginning of ice formation is clearly identified by the monitored sink (probe) temperature, and the time evolution of the ice front inside the tested material follows a typical power law (square root of time) of thermal diffusion phenomena.

In this paper, using the mentioned apparatus with the same experimental approach, the preliminary tests on the ex vivo bovine liver are presented. The choice of liver was driven by its physical characteristics, approximately homogeneous and isotropic. Results show the analogous behavior already found for water and agar-gel, about the onset of the beginning of the phase transition, and a similar maximum ice front progression measured in the case of agar versus the square root of the relative time.

In the experimental method, based on previous obtained results in the case of water [13], a linear relation between the heat removed per unit of area and the maximum ice front progression has been successfully verified in case of ex vivo bovine liver. Moreover, an attempt to determine the useful duration of an ablative treatment to avoid damage in the proximal anatomical structures (e.g. the phrenic nerve palsy) was made by splitting the heat removed by the probe in useful and useless components, where the former induces the ice formation inside the tissue, while the latter produces the cooling of the surrounding tissue only.

Figure 1. Schematic drawing of the experimental arrangement and a geometric sketch of the copper cold finger ( $h=25 \mathrm{~mm}, d=15 \mathrm{~mm})$, with the location of the thermocouples. The measurement vessel is of parallelepiped shape, square base of $43 \mathrm{~mm}$ on each side, and $30 \mathrm{~mm}$ high.

\section{Methods}

The arrangement employed in the experiments is described and validated in a previous paper [13], therefore the detailed description of the apparatus components is here omitted, and the schematic drawing in Figure 1 only recalls the main parts of the cooling system jointed to the measurement vessel with a symbolic representation of the tested material, and a geometric sketch of the copper cold finger, including main dimen- 
sions, with the location of the thermocouples.

The freezing effect is induced by a probe of appropriate shape named cold finger, whose top circular surface (about $1.77 \mathrm{~cm}^{2}$ ) makes contact with the material under test arranged inside the vessel, while its square base is thermally joint with a Peltier stack assembled with four modules, fed by separated power supplies, and cooled by a heat exchanger linked to a cold storage maintained at a low temperature by a chiller.

The adopted measurement technique named mirror image is described by Giaretto and Passerone [13]. With this experimental technique, thermal events inside the tested material are deduced from thermal measurements outside of the material itself. In this way, the probe (the cold finger in our case) is both a cooling device and a thermal sensor, and temperatures collected in some spots inside it make possible to obtain the thermal heat flux subtracted at the interface with the tested material, and the expected signal regarding the occurring change of phase.

Heat flux density subtracted at the mentioned cold interface was obtained assuming the one-dimensional transient heat diffusion for an isotropic and homogeneous medium (the cold finger), with the measured temperatures at $\mathrm{z}=0$ and $\mathrm{z}=-\mathrm{h}$ as Dirichlet boundary conditions at a given time t. The solution of the heat diffusion equation was obtained numerically [13].

Following this approach, the beginning of ice formation at the probe interface and its penetration inside the tested material can be identified by monitoring the probe temperature only. In previous experiments, these occurrences were verified on mimicked tissue, comparing the measured thermal response with data obtained by a digital camera. These experiments were possible because previous tested materials (both water and agar-gel) are transparent in the visible spectrum, and the ice growth history inside these were easily related with the thermal events measured at the interface.

After the onset of liquid-solid phase transition, the ice growth along the probe normal direction (the symmetrical axis of the cold finger in our case, i.e. the maximum penetration) follows a linear trend versus the square root of time, whose slope depends on both the material features (structure, composition, etc.), and the heat subtracted per unit area at the solid interface. In fact, a different slope depending on the shape of the ice volume inside the material structure were found for water and agar-gel, and a linear agreement was attained with

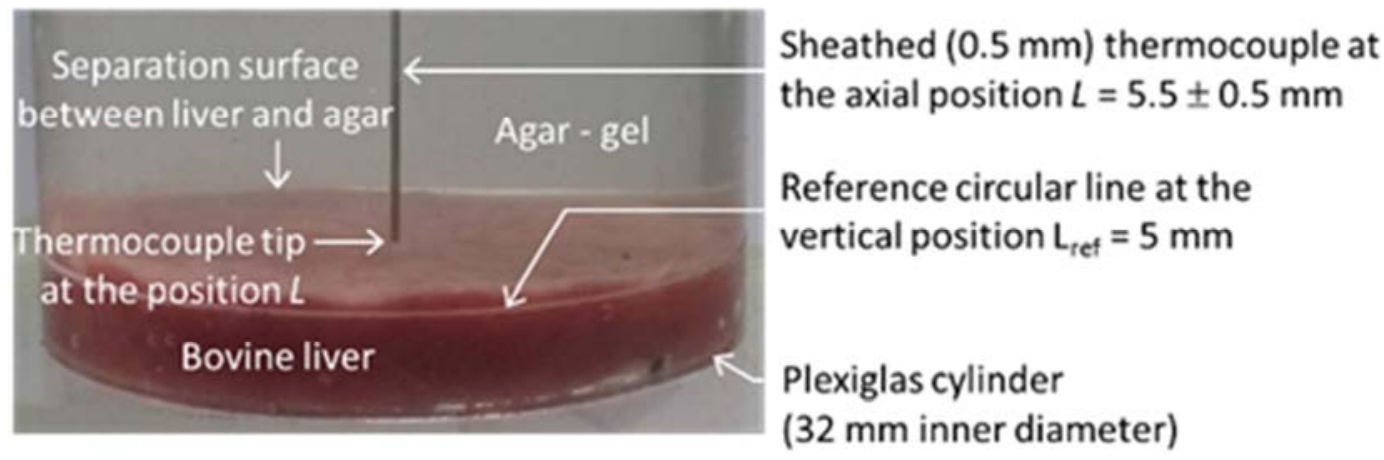

Figure 2. Picture of Sample \#1. The sample includes a layer of bovine liver (thickness $5.5 \pm 0.5 \mathrm{~mm}$ ), on which a thick layer of agar-gel is superimposed. the increasing ice volume and the heat removed per unit area in the case of water.

Consequently, the maximum ice penetration can be assumed proportional to the heat flux density subtracted by the probe. For example, denoting the heat rate subtracted per unit area as q, supposing that the ice has penetrated up to distance $\mathrm{z}$ from the cold interface after a given time interval $\Delta \mathrm{t}$, in case the heat subtracted is q', within the same time interval, the penetration of the ice should reach the position $z$ ' given by:

$$
z^{\prime}(\Delta t)=z(\Delta t) q^{\prime} / q
$$

The experimental methodology validated in previous experiments and summarized above is now employed for investigating the behavior of ex vivo bovine liver with the following targets:

identify the onset of ice formation at the probe-tissue interface with the help of temperatures measured at the probe only, and compare the obtained results with data found in the literature about a real cryoprobe application in actual clinical treatments;

verify in the case of ex vivo bovine liver the linear relationship between the maximum ice front penetration and the square root of time previously found in the case of water and agar-gel, and check the linear link between the heat flux removed per unit of surface and the maximum ice front penetration expressed by (1).

Two samples of the liver of a bovine obtained from a local animal processing facility have been produced with different shapes. The prepared liver samples have been stored in a small refrigerating cell maintained at about $6^{\circ} \mathrm{C}$, and before the apparent deterioration of liver samples occurred in about 48 hours, only two samples were employed in the experiments. The first, named Sample \#1 (whole), was made from a liver slice about $5 \mathrm{~mm}$ thick, prepared using the sharp disk of a slicer to obtain as flat and parallel surfaces as possible. From this layer, a cylindrical portion of bovine liver $32 \mathrm{~mm}$ in diameter was formed through a die-cutter. The slice thus prepared was then assembled at one end of an open cylindrical container made in Plexiglas, with the same internal diameter, and height suitable to contain an adequate portion of agar-gel of thickness at least equal to its diameter.

Sheathed $(0.5 \mathrm{~mm})$ thermocouple at

Reference circular line at the vertical position $\mathrm{L}_{\text {ref }}=5 \mathrm{~mm}$

\section{Plexiglas cylinder} (32 $\mathrm{mm}$ inner diameter) 


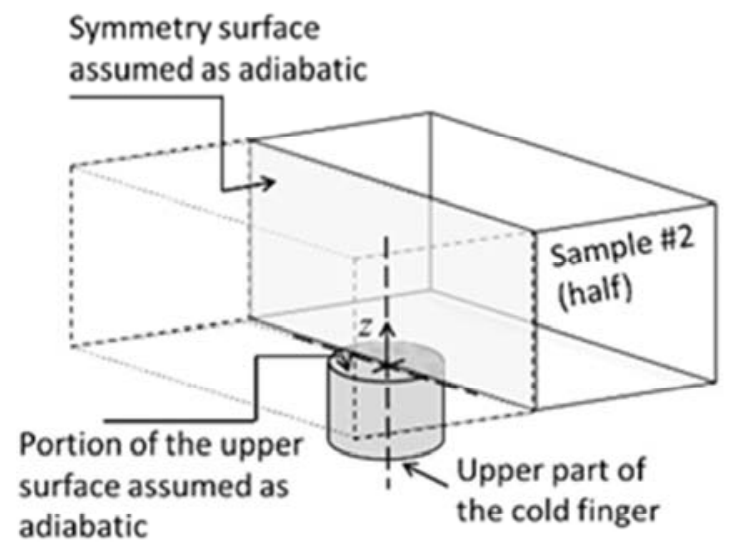

(a)

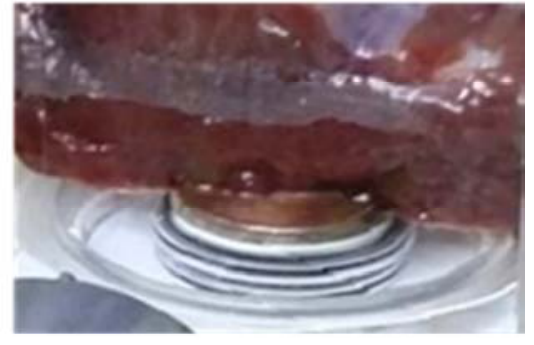

(b)

Figure 3. (a) Schematic representation of the arrangement inside the measurement vessel of the symmetric portion of Sample \#2. (b) Picture of the symmetry surface of Sample \#2, taken just before the test starts.

For this purpose, a solution in distilled water of $2 \%$ by weight of agar was prepared by heating and homogenizing the mixture at a constant temperature not higher than $40^{\circ} \mathrm{C}$. Taking care to minimize the temperature difference between the liver and the agar solution, the latter was directly filled over the liver in the free part of the cylinder, waiting for the complete gelification at room temperature.

The ex vivo tissue is the actual investigated material; the overlying agar layer is conceived to give an adequate separation from the external environment, and allows placing a temperature sensor (type $\mathrm{K}$ sheathed thermocouple, $0.5 \mathrm{~mm}$ in diameter) in contact with the upper surface of the liver along the vertical axis, as shown in Figure 2. The axial position $\mathrm{L}$ of the thermocouple has been determined with the help of the reference circular line engraved on the external surface of the Plexiglas cylinder, at the nominal distance $\mathrm{L}_{\mathrm{ref}}=5 \mathrm{~mm}$ from the liver-probe interface. Considering the irregularities of the liver upper surface, the tip of the thermocouple falls within an axial coordinate in a range between 5 and $6 \mathrm{~mm}$, therefore assuming $\mathrm{L}=5.5 \pm 0.5 \mathrm{~mm}$.
In this way, the temperatures $T_{I}$ and $T_{L}$ measured on the liver boundary, at the probe-tissue interface and axial position L (tissue-agar interface), respectively, allow discriminating some thermal effects inside the tissue layer, as discussed in the next section.

The second sample, named Sample \#2 (half), was made using a thick slice of the same bovine liver employed for the preparation of first the sample, approximately of parallelepiped shape, with dimensions similar to the measurement vessel in width and height, but half deep, as shown in Figure 3. This massive shape was chosen to take advantage of the axial-symmetry condition with which the freezing effect is generated inside the tested material. In fact, covering with the material only a half portion of the top circular surface of the cold finger, assuming as adiabatic both the uncovered part of the cold finger and the vertical free surface of the material itself, the thermal response inside the latter is expected to be equal to the thermal response resulting by covering the whole surface.

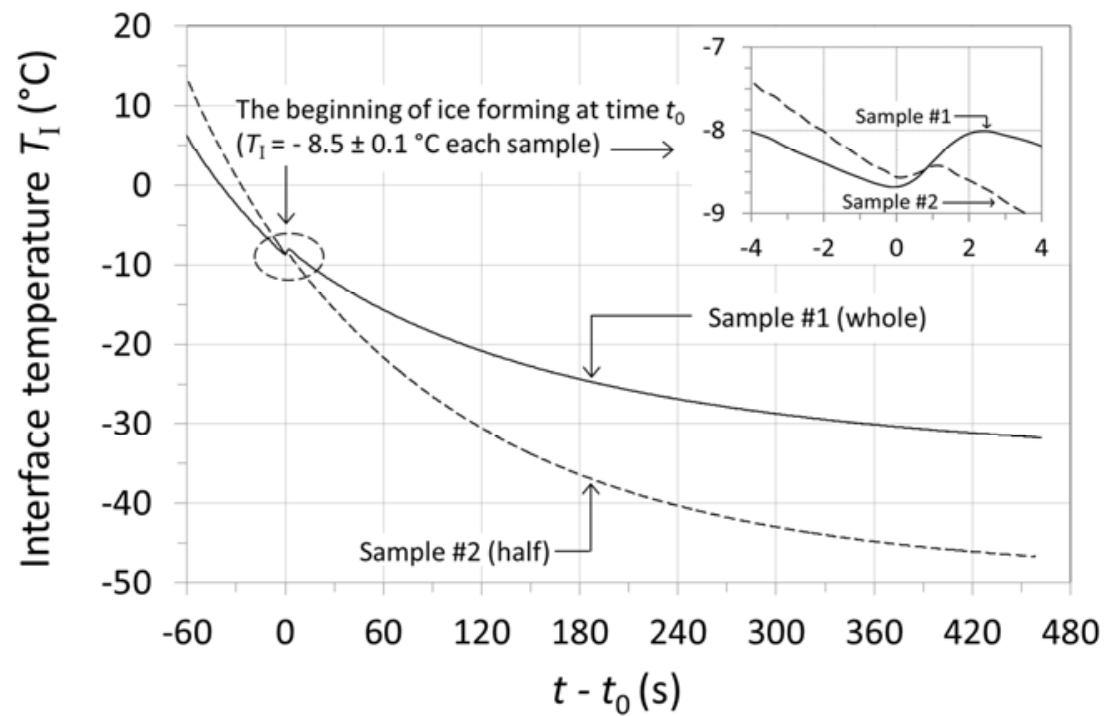

Figure 4. Temperatures measured at the top edge of the cold finger versus relative time, in the case of Sample \#1 (solid line) and Sample \#2 (dashed line); $t_{0}$ is the time at which the phase transition occurs. On the right side a magnification of temperature trends around the sudden ice trigger. 


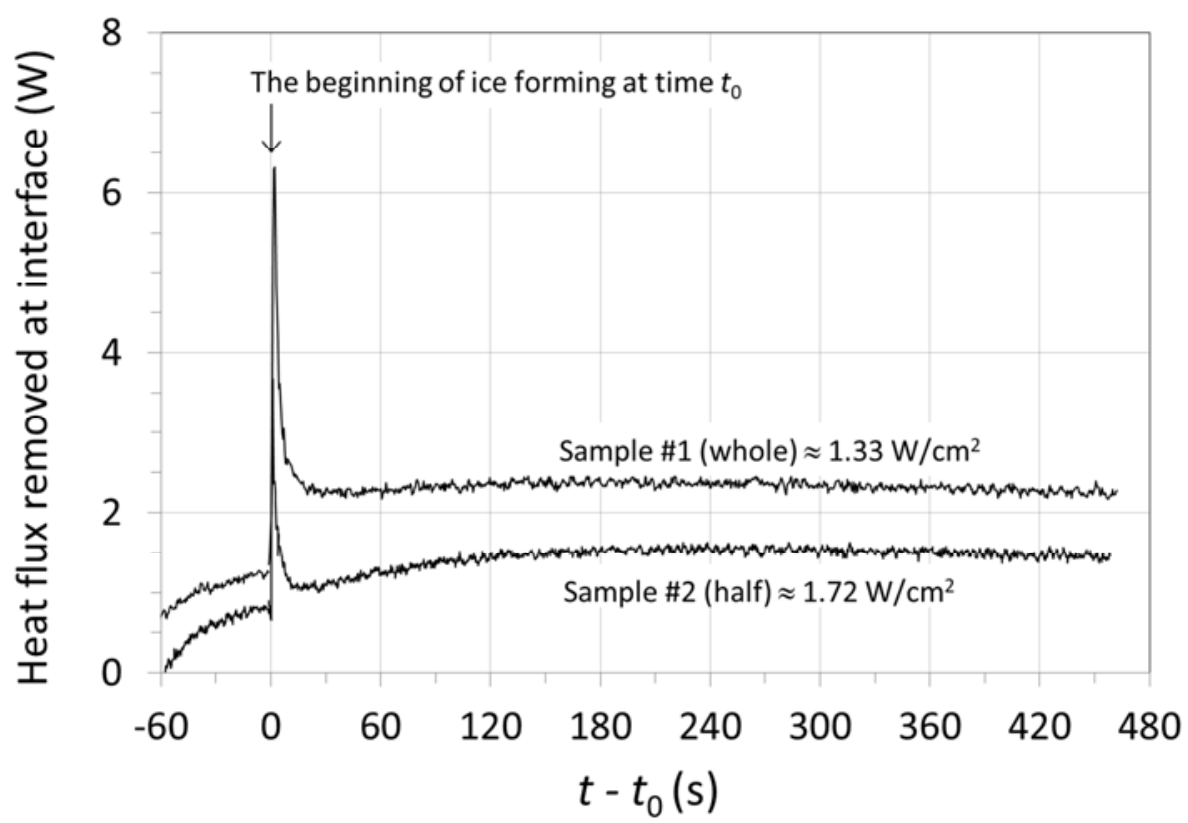

Figure 5. Removed heat flux versus relative time involving both samples; $t_{0}$ is the time at which the phase transition occurs. The displayed heat flux densities refer to the average values calculated after the beginning of ice formation, omitting the measured values close to the peak.

The adiabatic assumption for both free vertical surface of the sample and half circular surface of the cold finger is valid as a first approximation only. Despite some difficulties in positioning the biological sample on an actual half portion of the probe, and the lack of planarity of the vertical portion of the liver (because of the defective cut and its own weight), from the experimental point of view, this arrangement allows investigating with a digital camera the trigger of the phase transition and the propagation of the ice front inside the liver, as obtained for a transparent material.

\section{Results and Discussion}

In tests performed on both samples, the Peltier devices in stack were fed with the same set of electrical currents, each one independently in the range $1-5 \mathrm{~A}$.

Figure 4 and Figure 5 show the measured temperatures at the probe-tissue interface and the resulting heat flux subtracted for both samples versus the relative time $\left(t-t_{0}\right)$, respectively, where $t_{0}$ is the time at which the liquid-solid phase transition occurs inside the liver samples. The beginning of ice formation corresponds to a sudden release of heat (the peak of heat flux shown in Figure 5) that increases quite rapidly the sink (probe) temperature, as displayed in the enlargement on the top right of Figure 4. The heat flux densities q and q' displayed in the labels inside Figure 5 close to the graphs refer to their average values calculated after the ice formation, omitting the measured values close to the peaks. Note that the heat flux density for Sample \#2 is higher than the density for Sample \#1 because the area of contact with the probe is smaller.

The behavior found for the bovine liver perfectly matches what was previously observed in the case of water and agar-gel [13], confirming that the ice triggering in the frozen material can be identified by the measured temperature inside the probe. The thermodynamic state at which the phase transition occurs is also in this case supercooled, with an interface temperature of about $-8.5^{\circ} \mathrm{C}$, practically equal for both samples. Because the heat capacity of the cold finger is relatively high, and the heat released during the sudden phase change not so high, the measured temperature increment is quite small, in this case less than one degree Celsius.

Regarding the ability to detect the onset of ice formation, it is interesting to observe what happens by using an actual cryoballoon (Arctic Front, 28-mm diameter, Medtronic CryoCath LP, Pointe-Claire, Quebec, Canada) for a pulmonary vein isolation in patients with atrial fibrillation. For this purpose, as a useful example, the temperature data for the expanded gas leaving the cryoballoon, representative of successful and unsuccessful clinical treatments, have been deduced in a fairly accurate way from the graph reported by Fürnkranz et al. ([14], page 822), and displayed in Figure 6.

In the figure, continuous and dashed lines refer to successful and unsuccessful vein isolation respectively. Both temperature trends show a sudden increase in temperature that could refer to the mentioned onset of the phase transition. In this case the observed temperature increase is greater than that observed with the massive cold finger, since the thermal capacity of the probe is evidently smaller. In fact, by using a cryoballoon, the freezing effect is produced by throttling a small flow rate of a fluid (typically some tens of milligrams per second of $\mathrm{N}_{2} \mathrm{O}$ ), and the heat capacity depends on both the mass flow and its latent heat of vaporization. 


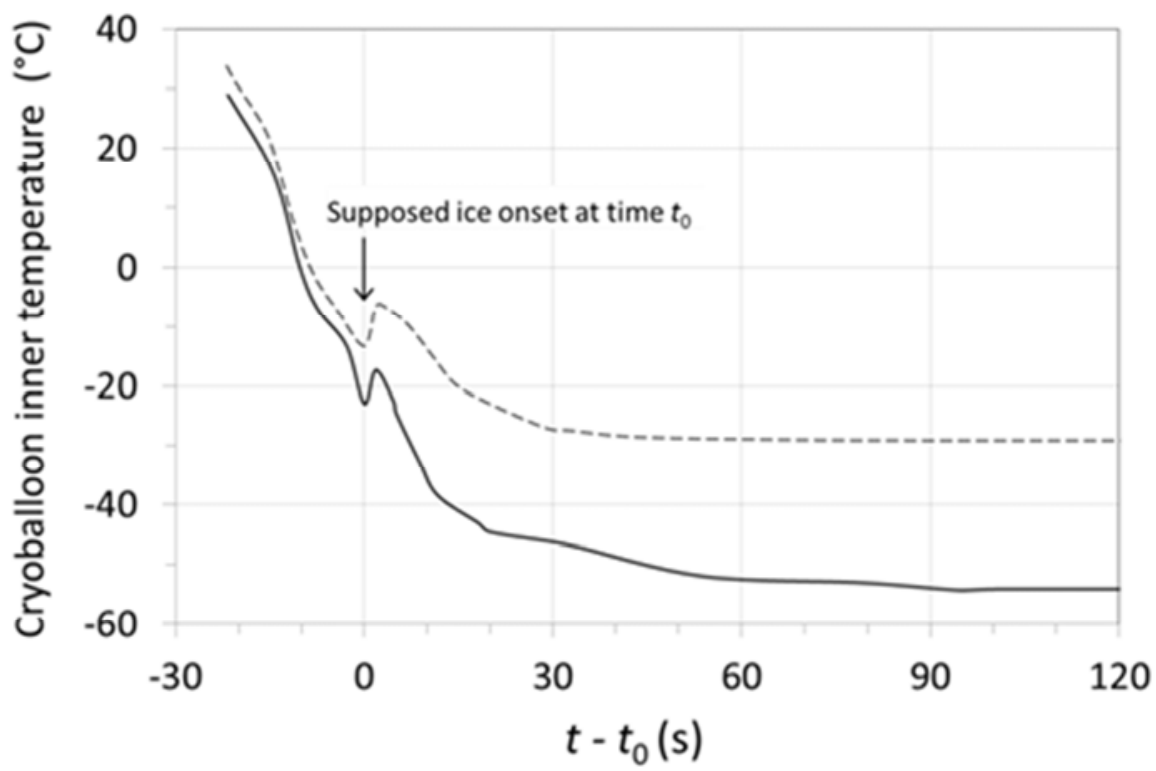

Figure 6. Temperature trends recorded by a cryoballoon (Arctic Front, 28-mm diameter, Medtronic CryoCath LP, Pointe-Claire, Quebec, Canada) in the case of successful (solid line) and unsuccessful (dashed line) pulmonary vein isolation. The temperature values versus time have been deduced from the graph reported reported by Fürnkranz et al. ([14], page 822).

However, it is possible to state that also for an actual cryogenic probe the ice triggering can be identified by the temperature of the sink, and a quick evaluation of the heat flux subtracted by the probe during ablation could be useful on predicting the effectiveness of a treatment.

In our experiments, the ice growth in both liver samples was identified in different ways. In the case of Sample \#2, arranged in the measurement vessel as shown in Figure 3, the ice growth evolution in time was easily detected on the observable symmetry surface by a standard digital camera, employing a digital image processing technique on the recorded frames at each time step $[13,15]$. Some frames recorded by the camera at different times after ice formation inside the tissue are shown in Table 1.

Table 1. Selected video frames from the onset of the phase transition $\left(t=t_{0}\right)$ in Sample \#2 and after various time intervals. The parameters in the data column are the relative time $\Delta t=t-t_{0}(\mathrm{~s})$, and the penetration distance $z(\mathrm{~mm})$.

Data
$\Delta \mathrm{t}=0, \mathrm{z}=0$
$\Delta \mathrm{t}=1, \mathrm{z}=0.20$
$\Delta \mathrm{t}=4, \mathrm{z}=0.61$
$\Delta \mathrm{t}=16, \mathrm{z}=1.31$
$\Delta \mathrm{t}=36, \mathrm{z}=2.00$
$\mathrm{t}=64, \mathrm{z}=2.66$

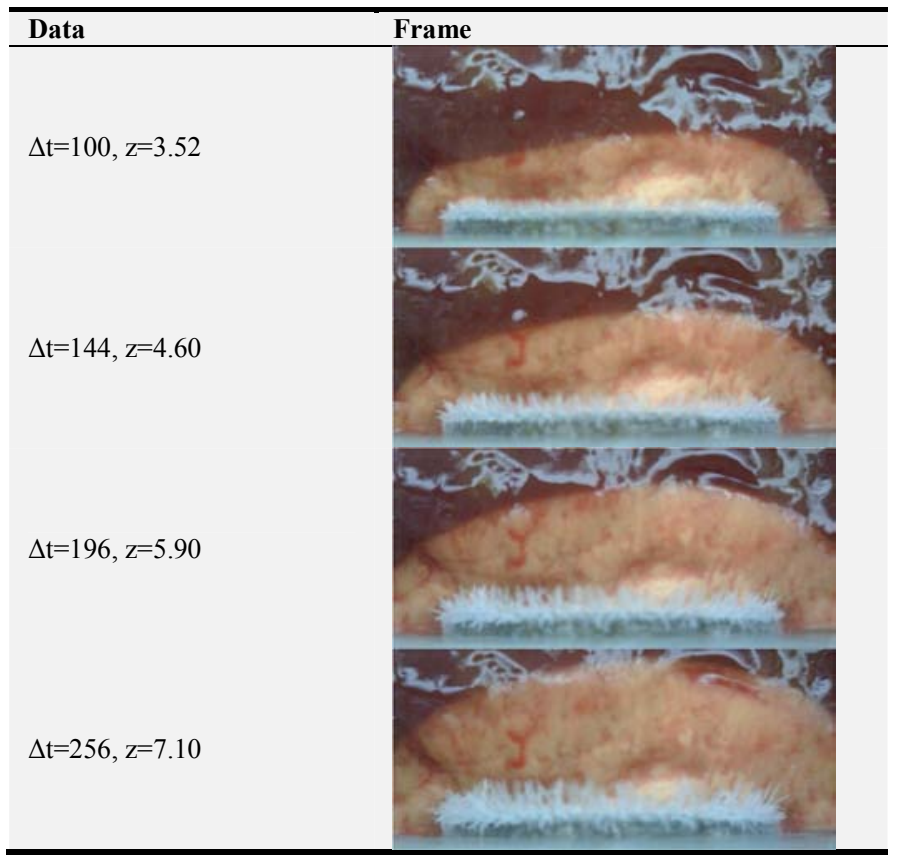

The selected frames in the table show an imperfect symmetry of the ice growth on the visible face of the liver due to previous mentioned non-planarity of this surface. Although this may seem improper, a good agreement with a linear growth of the ice front versus the square root of time was nevertheless found, as shown in the graph of Figure 6 discussed later. At the beginning the shape of the frozen portion of the liver is like the cold finger footprint; afterwards, the ice propagation inside the tissue becomes more symmetric, and its shape more closely reproduces the expected thermal diffusion in a homogenous and isotropic material. In the last images, due to the humidity of the air in the measuring vessel, ice needles on the uncovered cold interface are evident. By the estimated volume of ice formed, this leak of energy is evalu- 
ated around some percent.

In the case of Sample \#1, because the ice evolution inside the sample was not directly observable, the maximum ice front penetration was estimated by the ice growth assumption introduced with (1). A constant ratio between the different average values of heat flux density found in the experiments with Sample \#1 $\left(\mathrm{q}^{\prime}=1.33 \mathrm{~W} / \mathrm{cm}^{2}\right)$ and Sample \#2 $\left(\mathrm{q}=1.72 \mathrm{~W} / \mathrm{cm}^{2}\right)$ was adopted. So, at each relative time $\left(t-t_{0}\right)$ the maximum ice penetration $z^{\prime}\left(t-t_{0}\right)$ inside Sample \#1 was estimated proportionally $\left(q^{\prime} / q=1.33 / 1.72=0.773\right)$ to the measured penetration $\mathrm{z}\left(\mathrm{t}-\mathrm{t}_{0}\right)$ inside Sample \#2, where $\mathrm{t}_{0}$ is the ice onset time for each sample. Moreover, through the transparency of the agar layer arranged over the top of the liver slab forming Sample \#1, it was possible to identify the beginning time of the ice forming at the axial position $\mathrm{L}$, on the separation surface be- tween the materials. The arriving time has been detected manually, observing the light scattering near the axial position $\mathrm{L}$, estimating the beginning of ice formation in a time $\mathrm{t}-\mathrm{t}_{0}$ ranged between $248 \mathrm{~s}$ and $258 \mathrm{~s}$ (which corresponds to $\sqrt{ }\left(\mathrm{t}-\mathrm{t}_{0}\right)$ $\left.\approx 16 \mathrm{~s}^{1 / 2}\right)$. Furthermore, the complete freezing of the separation surface was detected at a time close to $305 \mathrm{~s}$. These results are shown in Figure 6, where the maximum ice front measured and estimated for both samples is reported versus the square root of relative time $\left(\mathrm{t}-\mathrm{t}_{0}\right)$, in addition to the beginning of ice forming at the separation surface of materials with its confidence region estimated from the conceived accuracy ranges for time and vertical position. In the figure, the ice growth measured in a previous experiment performed on the sole agar-gel is also shown.

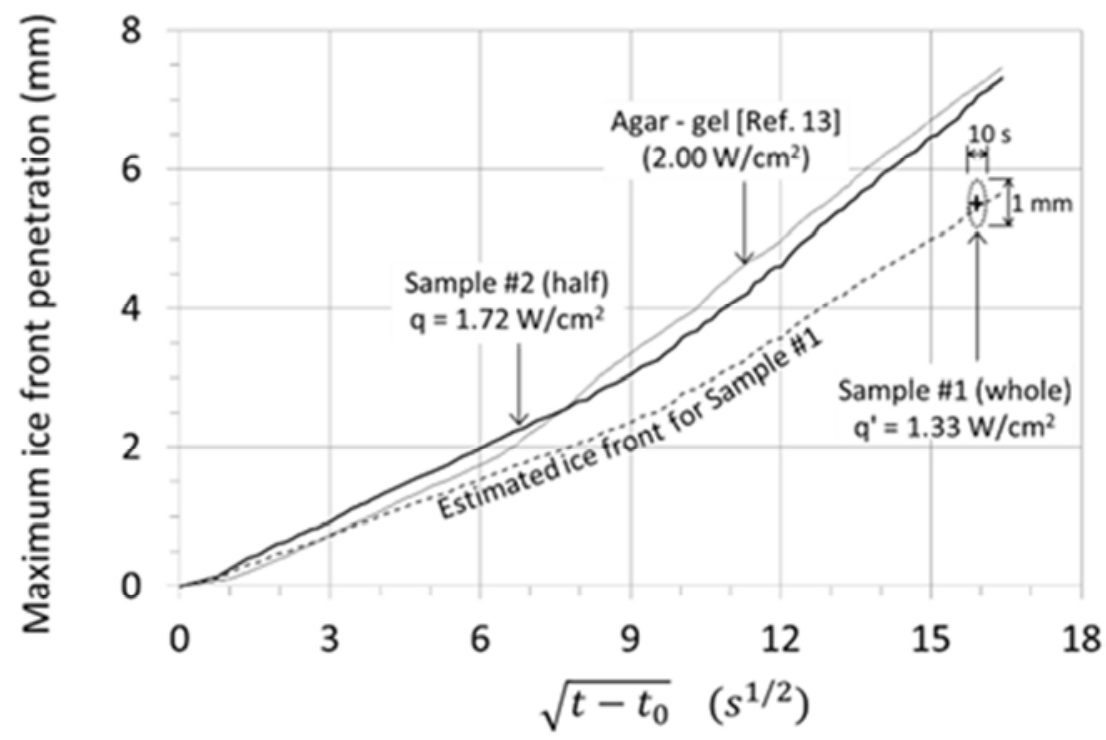

Figure 6. Maximum ice front penetration in the axial direction versus the square root of the relative time $t-t_{0}$, estimated by (1) in case of Sample \#1 (dashed line), measured in case of Sample \#2 (thick solid line) and agar-gel (thin solid line) from [13]. The penetration distance is measured from the edge of the cold-finger. Ice growth is linear versus the square root of relative time, and for Sample $\# 2$ the slope is approximately $0.332 \mathrm{~mm} / \mathrm{s}^{1 / 2}$ up to $t-t_{0} \approx 90 \mathrm{~s}$, and $0.595 \mathrm{~mm} / \mathrm{s}^{1 / 2}$ afterwards. The beginning of ice forming at the liver-agar separation surface of Sample \#1 is represented by the cross mark inside its confidence region (dotted elliptic line).

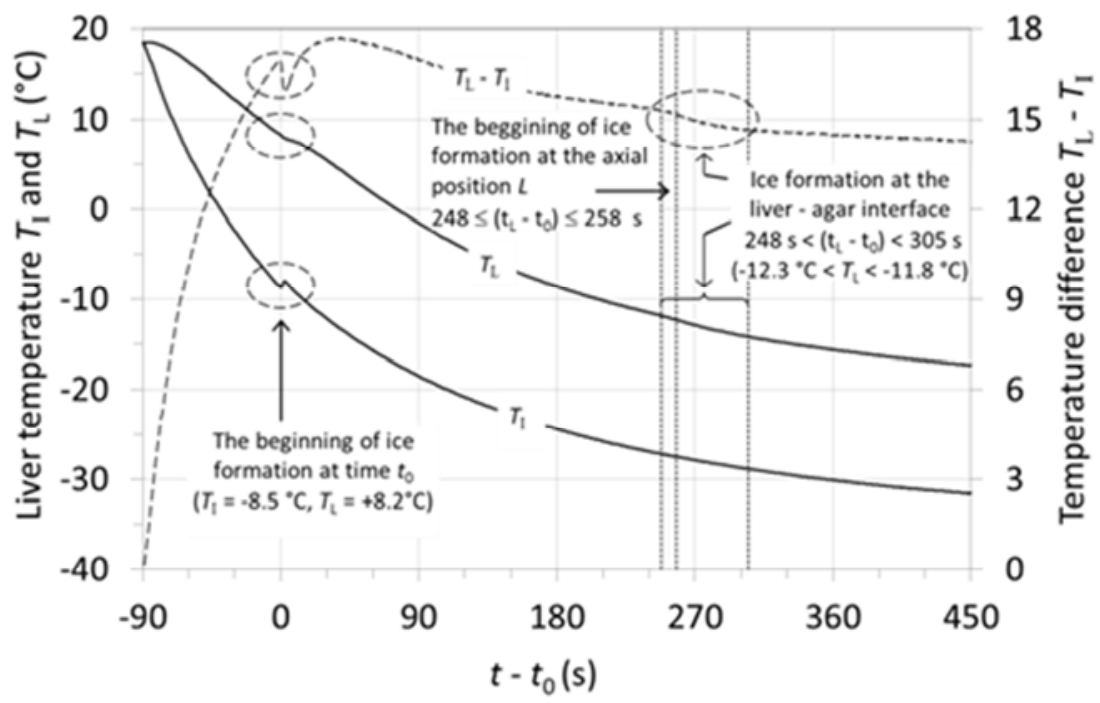

Figure 7. Measured temperatures $T_{I}$ and $T_{L}$ on Sample \#1 at the probe-liver and liver-agar interfaces (solid lines) versus the relative time t- $t_{0}$. Temperature difference across the liver layer (dashed line). 
The good agreement between the detected time for the beginning of ice forming at the axial position $\mathrm{L}(253 \pm 5 \mathrm{~s} ; 5.5 \pm$ $0.5 \mathrm{~mm}$ ) and its estimated growth with (1), confirms the suitability of the thermal diffusion process involving the ice growth inside materials and biological tissues with a significant water contents. Comparing the ice growth inside the liver with that previously measured in the agar, the similar behavior obtained with different heat flux densities allows hypothesizing that materials have an analogous structure, but a different water content, higher in the case of agar $2 \%$ by weight.

Regarding the temperature of ice forming inside the bovine liver layer of Sample \#1, the measured values obtained by the thermocouple placed at the vertical axial position L, on the separation surface between liver and agar, are shown in Figure 7 versus the relative time $\left(t-t_{0}\right)$. In the figure the temperature measured at the probe interface and the temperature difference across the whole liver layer are also shown.

The temperature at which the beginning ice is formed at the inner position $\mathrm{L}\left(\mathrm{T}_{\mathrm{L}} \approx-12^{\circ} \mathrm{C}\right)$ is lower than the temperature for the ice triggering at the probe-liver interface $\left(\mathrm{T}_{\mathrm{I}} \approx-8.5^{\circ} \mathrm{C}\right)$. When ice is forming at the probe interface, the liver temperature $T_{L}$ at position $L$ is widely greater than zero, and the onset of the phase transition produces a visible change in the temperature trend anywhere inside the liver layer. After the initial trigger, the ice forming inside the tissue does not produce evident signals traceable from the trend of the individual temperatures measured inside the material. It is interesting to note that the difference between the temperatures that delimit the liver layer shows a transition when the ice starts forming at the liver-agar interface, because of the different water content and the changing of thermal diffusion properties when the liquid-solid transition occurs.

Focusing on the behavior of the bovine liver layer of Sample \#1 after ice triggering, the heat flux subtracted by the probe produces the ice growth inside the tissue and the cooling of the neighboring medium. The ice transition and its growth inside the tissue are the expected useful effects, while the simple cooling of neighboring tissues may be useless or harmful for the proximal anatomical structures.

Despite the different thermal properties of the frozen and unfrozen states, no direct information on ice growth inside the liver can be obtained observing the trend versus time of the temperature measured at the layer interfaces. However, it is possible to evaluate some thermal contributions involving the heat conduction through the layer, the latent heat for ice formation and the energy spent for cooling the whole layer before and after ice formation. Considering the frozen and unfrozen properties of ex vivo liver found in the literature (see [16-17]), by using a simple thermal model in which the average properties of the whole layer are determined with a linear combination of frozen and unfrozen material portions, weighting up by the ice front propagation with (1), referring to a unit of probe surface it is possible to write:

$$
\varphi_{\mathrm{I}}=\left|\varphi_{\mathrm{L}}\right|+\left|\varphi_{\mathrm{C}}\right|+|\mathrm{du} / \mathrm{dt}|
$$

where, $\varphi_{\mathrm{I}}$ is the absolute value of the calculated specific heat flux subtracted at the probe interface, $\left|\varphi_{0}\right|$ the latent heat of ice forming inside the layer, depending on its growth rate, $\left|\varphi_{C}\right|$ the contribution due to the neighboring medium, depending on the measured temperature difference $\mathrm{T}_{\mathrm{L}}-\mathrm{T}_{\mathrm{I}}$, and $|\mathrm{du} / \mathrm{dt}|$ the rate of variation of the average internal energy of the layer depending on both its average temperature and the frozen-unfrozen liver portion.

The interest is to highlight the part $\left|\varphi_{L}\right|$ of the heat flux subtracted by the probe devoted to ice formation with respect to the remaining part $\left|\varphi_{\mathrm{C}}\right|+|\mathrm{du} / \mathrm{dt}|$ spent for cooling the liver layer and the neighboring medium. Introducing the ratio $f$ as:

$$
\mathrm{f}=\left|\varphi_{\mathrm{L}}\right| / \varphi_{\mathrm{I}}=1-\left(\left|\varphi_{\mathrm{C}}\right|+|\mathrm{du} / \mathrm{dt}|\right) / \varphi_{\mathrm{I}}
$$

its trend versus the relative time $\left(\mathrm{t}-\mathrm{t}_{0}\right)$ is reported in Figure 8.

In the figure, at the ice trigger the estimated ratio $\mathrm{f}$ is very close to the unitary value and decreases quite rapidly afterwards. At the beginning of the phase transition the energy subtracted by the probe is primarily addressed to ice formation and only marginally to cooling the neighboring material, on the converse with increasing time.

This behavior estimated for Sample \#1 agrees with the ice progression measured for Sample \#2 (compare data and frames shown in Table 1), and agrees with the estimated ice drift velocity in Sample \#1 depicted in Figure 8. The penetration rate goes from $0.2 \mathrm{~mm} / \mathrm{s}$ at the very beginning to 0.02 $\mathrm{mm} / \mathrm{s}$ after about one minute, and then remains quite constant with only a slight decrease. On this basis, to improve the cryoablation effectiveness it could be useful to increase the heat flux subtracted per unit of area, in order to improve the beginning of ice formation within the tissue contacting the probe, and decrease the ablation duration, to reduce the possible damages from cooling in the proximal anatomic structures.

\section{Conclusions}

The performed experimental investigation on samples of ex vivo bovine liver allows highlighting some essential points.

The ice formation at the probe-liver interface produces a temperature perturbation inside the tested material similar to that previously found for water and agar-gel. As expected, such thermal effect can be indirectly identified by temperatures measured with the probe only. In particular, the results carried out in the experiments agree with data found in literature about a real cryoprobe application in case of pulmonary vein isolation, for which the monitored sink temperature gives a useful signal for identifying the beginning of ice formation. 


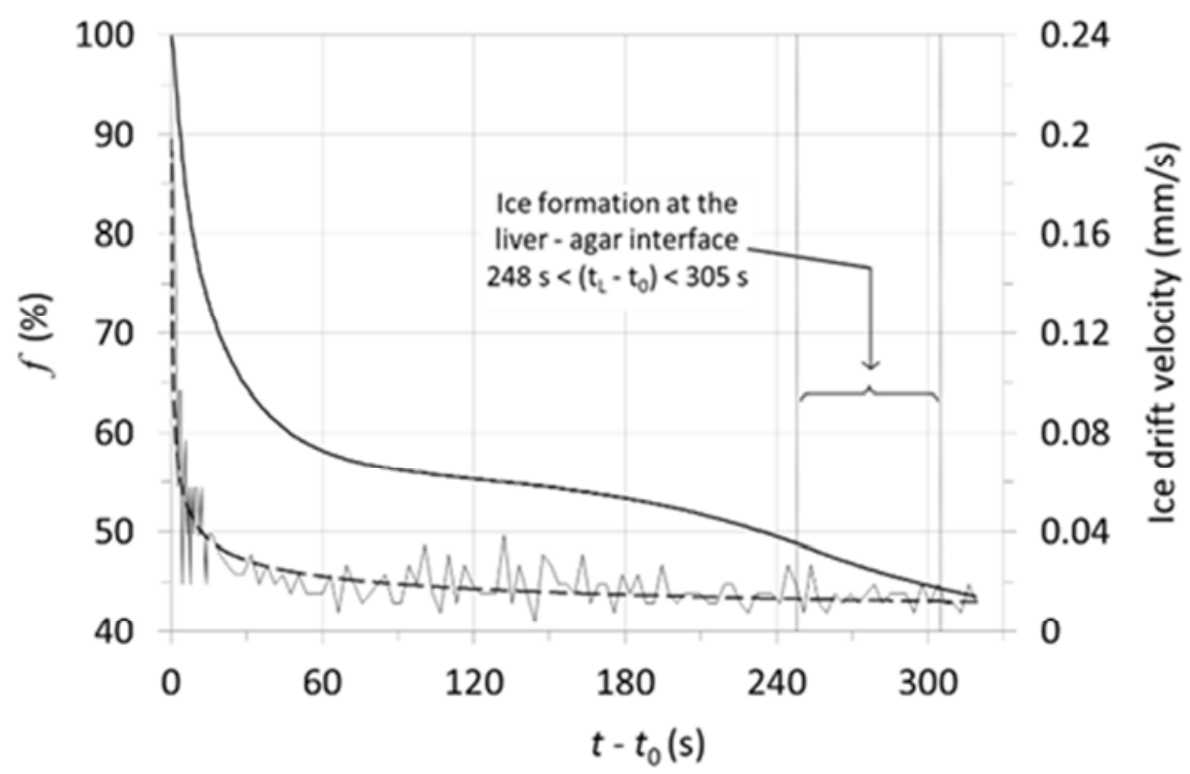

Figure 8. Fraction of the total heat removed spent for the ice forming inside Sample \#1 (thick solid line) versus the relative time t-to. Estimated ice drift velocity in Sample \#1 (thin solid line) and its regression (dashed line).

After the ice starts at the probe interface, its penetration inside the tested material depends on the rate of heat removed by the probe, and previous investigations show that its growth in normal direction (maximum penetration) can be assumed linear with the heat subtracted per unit of surface. This behavior was confirmed in the case of liver, for which the arriving time of ice at a given inner position matches the one estimated by the theoretical model, through which is affirmed that the ice growth is linear also with the square root of time, and the penetration distance at a given time can be proportionally conformed to heat removed per unit of surface.

In summary, monitoring the sink temperature and the heat rate subtracted by the probe, an effective estimation of the ice penetration inside the tissue may be provided, especially in first moments after the ice formation, in which the energy taken off by the probe is primarily spent for removing the latent heat of solidification rather than cooling the surrounding medium. In the case of bovine liver few tens of seconds are sufficient to reach a maximum penetration distance of about 2 $\mathrm{mm}$ for the ice front inside the tissue.

This methodology may be applied to current cryoprobes, which already provide some of the physical quantities required in the presented technique, to drive cryoablation in order to enhance its effectiveness, avoiding prolonged inefficient cooling.

\section{References}

[1] Mazur, P. (1984). Freezing of living cells: Mechanisms and implications. American Journal of Physiology - Cell Physiology, 16, C125-C142.

[2] Choi, J., \& Bischof, J. C. (2011). Cooling rate dependent biophysical and viability response shift with attachment state in human dermal fibroblast cells. Cryobiology, 63, 285 -291 .
[3] Toner, M., Cravalho, E., Stachecki, J., Fitzgerald, T., Tompkins, R., Yarmush, M., \& Armant, D. (1993). Nonequilibrium freezing of one-cell mouse embryos. Membrane integrity and developmental potential. Biophysical Journal, 64, 1908-1921.

[4] Pegg, D. E., \& Diaper, M. P. (1988). On the mechanism of injury to slowly frozen erythrocytes. Biophysical Journal, 54, $471-488$.

[5] Muldrew, K., \& McGann, L. (1990). Mechanisms of intracellular ice formation. Biophysical Journal, 57, 525 - 532.

[6] Muldrew, K., \& McGann, L. (1994). The osmotic rupture hypothesis of intracellular freezing injury. Biophysical Journal, $66,532-541$.

[7] Han, B., \& Bischof, J. C. (2004). Direct cell injury associated with eutectic crystallization during freezing. Cryobiology, 48 , 8-21.

[8] Acker, J. P., Elliott, J. A., \& McGann, L. E. (2001). Intercellular ice propagation: Experimental evidence for ice growth through membrane pores. Biophysical Journal, 81, 1389 - 1397.

[9] Balasubramanian, S., Bischof, J., \& Hubel, A. (2006). Water transport and iif parameters for a connective tissue equivalent. Cryobiology, 52, 62-73.

[10] Weng, L., Tessier, S. N., Swei, A., Stott, S. L., \& Toner, M. (2017). Controlled ice nucleation using freeze-dried pseudomonas syringae encapsulated in alginate beads. Cryobiology, $75,1-6$.

[11] Gaita, F., Riccardi, R., Caponi, D., Shah, D., Garberoglio, L., Vivalda, L., Dulio, A., Chiecchio, A., Manasse, E., \& Gallotti, R. (2005). Linear cryoablation of the left atrium versus pulmonary vein cryoisolation in patients with permanent atrial fibrillation and valvular heart disease. Circulation, 111, 136-142.

[12] Gaita, F., Caponi, D., Scaglione, M., Montefusco, A., Corleto, A., Di Monte, F., Coin, D., Di Donna, P., \& Giustetto, C. (2008) Long-term clinical results of 2 different ablation strategies in patients with paroxysmal and persistent atrial fibrillation. Circulation: Arrhythmia and Electrophysiology, 1, 269-275. 
[13] Giaretto, V., \& Passerone, C. (2017). Mirror image technique for the thermal analysis in cryoablation: Experimental setup and validation. Cryobiology, 79, $56-64$.

[14] Fürnkranz, A., Köster, I., Chun, K. J., Metzner, A., Mathew, S., Konstantinidou, M., Ouyang, F., \& Kuck, K. H. (2011). Cryoballoon temperature predicts acute pulmonary vein isolation. Heart Rhythm, 8, $821-825$.

[15] Gonzalez, R. C., \& Woods, R. E. (2006). Digital Image Processing ( $3^{\text {rd }}$ Edition). Upper Saddle River, NJ, USA: Prentice-Hall, Inc.

[16] Kim, C., O’Rourke, A. P., Will, J. A., Mahvi, D. M., \& Webster, J. G. (2008). Finite-element analysis of hepatic cryoablation around a large blood vessel. IEEE Transactions on Biomedical Engineering, 55, 2087-2093.

[17] Chan, J. Y., \& Ooi, E. H. (2016). Sensitivity of thermophysiological models of cryoablation to the thermal and biophysical properties of tissues. Cryobiology, 73, $304-315$.

[18] Matta, M., Anselmino, M., Ferraris, F., Scaglione, M., Gaita, F. (2018). Cryoballoon vs. radiofrequency contact force ablation for paroxysmal atrial fibrillation: a propensity score analysis. J Cardiovasc Med, 19(4), $141-147$.

[19] Matta, M., Anselmino, M., Scaglione, M., Vitolo, M., Ferraris, F., Di Donna, P., Caponi, D., Castagno, D., Gaita, F. (2017).
Cooling dynamics: a new predictor of long-term efficacy of atrioventricular nodal reentrant tachycardia cryoablation. J Interv Card Electrophysio, 48(3), 333 - 341.

[20] Ting-Yung, C., Li-Wei, L., Abigail, L., Yenn-Jiang, L., Shih-Lin, C., Yu-Feng, H., Fa-Po, C., Tze-Fan, C., Nan, L., Ta-Chuan, T., Chin-Yu, L. (2018). The importance of Extra-Pulmonary Vein Triggers and Atypical Atrial Flutter in Atrial Fibrillation Recurrence After Cryoablation: Insights from Repeat Ablation Procedures. J Cardiovasc Electrophysiol. doi: 10.1111/jce. 13741.

[21] Larnier, L., Badenco, N., Thuillot, M., Bravetti, M., Gandjbakhch, E., Duthoit, G. (2018). Comparison of incidences of pulmonary vein stenosis between radiofrequency and cryoablation in atrial fibrillation ablation. Rhythmology and stimulation, 10(1), $89-90$.

[22] Marrouche, N. F., Brachmann, J., Andresen, D., Siebels, J., Boersma, L., Jordaens, L., Merkely, B., Pokushalov, E., Sanders, P., Proff, J., Schunkert, H., Christ, H., Vogt, J., Bänsch, D. (2018). CASTLE-AF Investigators. Catheter Ablation for Atrial Fibrillation with Heart Failure. N Engl J Med., 378(5), $417-427$.

[23] de Asmundis, C., Chierchia, G. B., La Meir, M. (2018). Extracardiac ice formation during CoolLoop cryoablation of atrial fibrillation. Pacing Clin Electrophysiol. 41, 1264 - 1265. 